\title{
Automated Classification of Quasars and Stars
}

\author{
Yanxia Zhang ${ }^{1}$, Yongheng Zhao ${ }^{1}$, and Hongwen Zheng ${ }^{2}$ \\ ${ }^{1}$ National Astronomical Observatories, Chinese Academy of Sciences, Beijing 100012, China \\ Email: zyx@lamost.org \\ ${ }^{2}$ North China Electronic Power University, Beijing, 102206, China \\ Email: zyx300@sohu.com
}

Keywords. astronomical databases: miscellaneous, catalogs, methods: data analysis, methods: statistical

We investigate selection and weighting of features by applying a random forest algorithm to multiwavelength data. Then we employ a $k$-nearest neighbor method to distinguish quasars from stars. We then compare the performance of this approach based on all features, weighted features, and selected features. We find that the $k$-nearest neighbor approach combined with random forests effectively separates quasars from stars.

The sample we used was cross-identified from different survey catalogs, i.e., the SDSS DR5, FIRST, and USNO-B1.0 catalogs. This yielded as sample of 6,479 quasars and 785 stars.

A random forest approach was used to compute a weight for each attribute which allows us to select the most important attributes. We used the $k$-nearest neighbor approach to discriminate between quasars and stars and the results for three different variants are shown in Table 1 . The accuracy for quasar selection is above $98 \%$ for all three variants, but the classification of stars is not as good. The overall accuracy is better than $89 \%$ and in the best case the total accuracy is $94.93 \%$. Table 1 also shows that the performance with weighted features or selected features is slightly better than that with all features. As a consequence, if we have many input features, we generally need selection or weighting of features before we begin $k$-NN model building.

Table 1. Separate quasars and stars by $k$-NN.

\begin{tabular}{|c|c|c|c|c|c|}
\hline Sample |All & Features & | Weighted & Features & Selected & Features \\
\hline classified $\downarrow$ known $\rightarrow \mid$ quasars & stars & | quasars & stars & | quasars & stars \\
\hline quasars 6440 & 705 & 6373 & 345 & 6373 & 262 \\
\hline stars $\mid 39$ & 80 & 106 & 440 & $\mid 106$ & 523 \\
\hline Accuracy $(\%) \mid 99.4 \pm 0.4$ & $10.2 \pm 2.3$ & | $98.4 \pm 0.4$ & $56.0 \pm 5.1$ & | $98.4 \pm 0.6$ & $66.6 \pm 5.3$ \\
\hline Total accuracy $(\%) \mid 89.76 \pm 0.55$ & & | $93.79 \pm 0.68$ & & | $94.93 \pm 0.86$ & \\
\hline
\end{tabular}

\section{Acknowledgements}

This work has been funded by the National Natural Science Foundation of China under Grant Nos. 90412016 and 10778724, and by 863 project under Grant No. 2006AA01A120. 